\title{
Using Physics to Speed up Tissue Engineering
}

\author{
Researchers have proposed and tested a new method that could speed up \\ bioprinting, a promising technique for fabricating organs for transplants. \\ By Sophia Chen
}

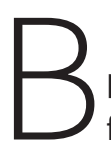

ioprinting-the 3D printing of human tissues-holds potential for producing tissues and organs

for transplants. In this technology, a machine dispenses small segments of bioinks-materials made of cell aggregates or microtissues. When placed next to each other, the segments fuse and self-assemble into sturdier tissue structures. However, because the self-assembly process is slow, tissues assembled using a patient's own cells cannot be printed in urgent clinical settings. Guided by a physics-based microscopic model, Ashkan Shafiee of Wake Forest School of Medicine, North Carolina, and colleagues have devised a new bioprinting method that accelerates tissue self-assembly [1].

Their method, based on bioink fusion, is an iterative process in which cells in one printed microtissue break off to develop new chemical bonds with an adjacent tissue. From a macroscopic perspective, this microscopic bond formation resembles a closing zipper. The researchers designed their method by harnessing a model prediction-that the bioinks would fuse faster when subjected to an external force. The model allowed

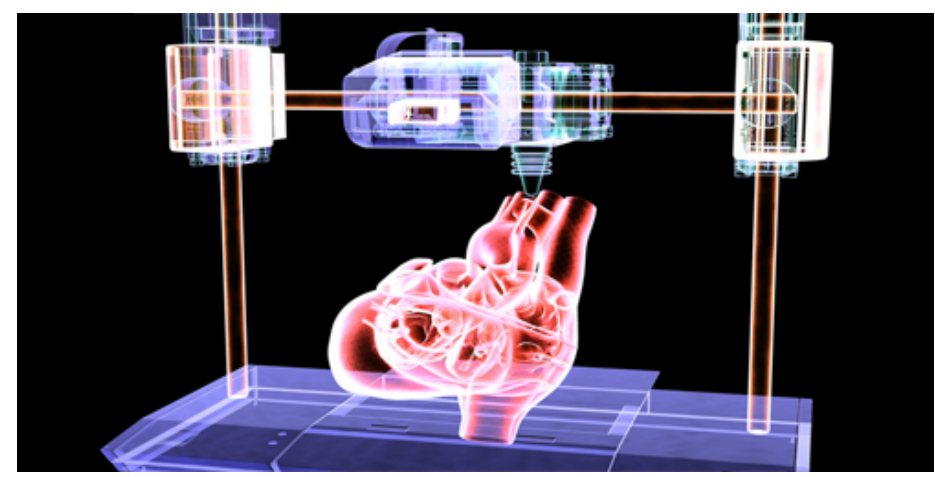

Credit: iStock.com/Devrimb them to optimize the magnitude of the force to accelerate bioink fusion without damaging existing cells.

In experiments, the team created a force with the desired magnitude by printing the bioinks into semicircular-shaped grooves on a gel platform. The grooves' geometry produced a rotational torque and other mechanical forces that caused the bioink segments to press against each other. By imaging the structural evolution of the fusing bioinks, they found that fusion occurred 3 times faster in the grooves than it did on a flat platform. The researchers are currently developing a bioprinter in which the forces on the bioinks can be finely tuned.

Sophia Chen is a freelance science writer based in Columbus, Ohio.

\section{REFERENCES}

1. A. Shafiee et al., "Acceleration of tissue maturation by mechanotransduction-based bioprinting," Phys. Rev. Research 3, 013008 (2021). 